\title{
Dual task effects on story retell for participants with moderate, mild, or no aphasia: Quantitative and qualitative findings
}

\author{
Tyson G. Harmon \\ Brigham Young University, tyson_harmon@byu.edu \\ Adam Jacks \\ University of North Carolina at Chapel Hill \\ Katarina L. Haley \\ University of North Carolina at Chapel Hill \\ Antoine Bailliard \\ University of North Carolina at Chapel Hill \\ Follow this and additional works at: https://scholarsarchive.byu.edu/facpub \\ Part of the Communication Sciences and Disorders Commons
}

\section{Original Publication Citation}

Harmon, T. G., Jacks, A., Haley, K. L., \& Bailliard, A. (2019). Dual-task effects on story retell for participants with moderate, mild, or no aphasia: Quantitative and qualitative findings. Journal of Speech, Language, and Hearing Research, 62(6), 1890-1905. https://doi.org/10.1044/

2019_JSLHR-L-18-0399

\section{BYU ScholarsArchive Citation}

Harmon, Tyson G.; Jacks, Adam; Haley, Katarina L.; and Bailliard, Antoine, "Dual task effects on story retell for participants with moderate, mild, or no aphasia: Quantitative and qualitative findings" (2019). Faculty Publications. 3250.

https://scholarsarchive.byu.edu/facpub/3250

This Peer-Reviewed Article is brought to you for free and open access by BYU ScholarsArchive. It has been accepted for inclusion in Faculty Publications by an authorized administrator of BYU ScholarsArchive. For more information, please contact ellen_amatangelo@byu.edu. 
Dual task effects on story retell for participants with moderate, mild, or no aphasia: Quantitative and qualitative findings

\author{
Tyson G. Harmon ${ }^{\mathrm{a}}$ \\ Adam Jacks \\ Katarina L. Haley ${ }^{b}$ \\ Antoine Bailliard ${ }^{\mathrm{c}}$
}

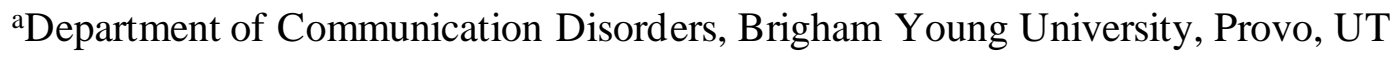

${ }^{b}$ Division of Speech and Hearing Sciences, Department of Allied Health Sciences, University of

North Carolina, Chapel Hill, NC

${ }^{\mathrm{c} D i v i s i o n}$ of Occupational Science and Occupational Therapy, Department of Allied Health

Sciences, University of North Carolina, Chapel Hill, NC

RUNNING HEAD: DUAL TASK EFFECTS ON STORY RETELL IN APHASIA

Please address correspondence to:

Tyson G. Harmon

Department of Communication Disorders

158 John Taylor Building

Brigham Young University

Email: Tyson_Harmon@ byu.edu

Telephone: 801-422-1251

Keywords: aphasia, cognition, communication, stroke, fluency, executive functions 


\begin{abstract}
Purpose: To determine dual task effects on content accuracy, delivery speed, and perceived effort during narrative discourse in people with moderate, mild, or no aphasia and to explore subjective reactions to retelling a story with a concurrent task.

Method: Two studies (one quantitative and one qualitative) were conducted. In study 1, participants with mild or moderate aphasia and neurotypical controls retold short stories in isolation and while simultaneously distinguishing between high and low tones. Story retell accuracy (speech productivity and efficiency), speed (speech rate, repetitions, and pauses), and perceived effort were measured and compared. In study 2, participants completed semistructured interviews about their story retell experience. These interviews were recorded, transcribed orthographically, and coded qualitatively using thematic analysis.

Results: The dual task interfered more with spoken language of people with aphasia (PWA) than controls but different speed-accuracy trade-off patterns were noted. Participants in the moderate aphasia group reduced accuracy with little alteration to speed, whereas participants in the mild aphasia group maintained accuracy and reduced their speed. Participants in both groups also reported more negative emotional and behavioral reactions to the dual task condition than their neurotypical peers. Intentional strategies for coping with the cognitive demands of the dual task condition were only reported by participants with mild aphasia.

Conclusion: The findings suggest that while communicating with a competing task is more difficult for PWA than neurotypical controls, participants with mild aphasia may be better able to cope with cognitively demanding communication situations than participants with moderate aphasia.
\end{abstract}




\section{Introduction}

Due to common distracting stimuli, everyday communication situations present greater demands on attention than quiet clinic environments. People with aphasia (PWA) have reported examples of these situations such as living in a city with a lot of background noise (Dalemans, de Witte, Wade, \& van den Heuvel, 2010, p. 545), "people talking or music and singing in the background" (Baylor, Burns, Eadie, Britton, \& Yorkston, 2011, p. 278), and "people talking all at once" (Parr, 2001, p. 276). They have also emphasized the challenge of communicating in these situations (Baylor et al., 2011; Dalemans et al., 2010; Garcia, Barrette, \& Laroche, 2000; Le Dorze, Salois-Bellerose, Alepins, Croteau, \& Hallé, 2014; Parr, 2001). Because of its relevance to everyday communication in aphasia, we sought to understand how attentional demands impact spoken language and the communication experience for PWA.

The role of attention in aphasia has been of interest for decades (e.g., Hula \& McNeil, 2008; Kriendler \& Fradis, 1968; McNeil, Odell, \& Tseng, 1991; Murray, 2002). Response to complex attentional demands are commonly tested using a dual task paradigm wherein participants are asked to process two sources of information concurrently. To be successful, they must allocate their attentional resources effectively across competing task demands (Hula \& McNeil, 2008; Kurland, 2011; Navon \& Miller, 2002). Numerous studies have demonstrated that performance is lower for participants with aphasia than for neurotypical peers (e.g., Hula, McNeil, \& Sung, 2007; LaPointe \& Erickson, 1991; Murray, 2000; Murray, Holland, \& Beeson, 1998; Tseng, McNeil, \& Milenkovic, 1993). For example, PWA have shown interference on both tasks when identifying a target word among foils and simultaneously sorting cards (LaPointe \& Erickson, 1991). Similarly, decreased performance has been demonstrated when 
semantic judgment and lexical decision tasks are presented simultaneously or concurrent with a tone discrimination task (Murray, Holland, \& Beeson, 1997b).

Although most of the dual task literature in aphasia has focused on auditory perception, interference has also been shown during spoken language production. This includes decreased accuracy and slower response times on phrase completion tasks when simultaneously distinguishing between two tones (Hula et al., 2007; Murray, 2000). Similarly, when tone discrimination was paired with a picture description task, participants with mild aphasia decreased speech productivity and efficiency and increased word-finding errors whereas neurotypical controls displayed no difference in spoken language performance (Murray et al., 1998). Further illustrating the attentional cost, the dual task in all these studies interfered not only with spoken language for PWA but also the secondary tone discrimination task.

Whereas spoken language interference has been primarily studied through content accuracy, both accuracy and speed have been considered for second ary tone discrimination tasks (Murray, 2000; Murray et al., 1998). When dividing their attention between speech production and tone discrimination, neurotypical controls increased their response time on the tone discrimination task in order to maintain high accuracy. PWA, on the other hand, were both slower and less accurate in tone discrimination (Murray, 2000; Murray et al., 1998). Given the dual task effects on speed-accuracy relationships during secondary task performance and the importance of both content and fluency during speech production, it would be valuable to better understand dual task effects on delivery speed and content accuracy in spoken language.

Another important consideration is how PWA respond subjectively to communication situations that tax their attentional system. This has been measured quantitatively using selfreport and qualitatively through semi-structured interviews. Self-ratings of effort may have 
implications for cognitive resource allocation (greater perceived effort would indicate more attentional resources being allocated to the task; Clark \& Robin, 1994). Several studies from the aphasia and stuttering literature have shown greater perceived effort as task demands increase (Clark \& Robin, 1994; Ingham, Bothe, Jang, Yates, \& Cotton, 2009; Ingham, Warner, Byrd, \& Cotton, 2006; Murray, Holland, \& Beeson, 1997a). The two studies specific to aphasia measured "sense of effort" and self-rated difficulty during lexical decision tasks. While increased task demands led to higher ratings, no differences were found between aphasia and control groups (Clark \& Robin, 1994; Murray et al., 1997a). Along with rating their effort as greater in a dual task condition, PWA recognize environmental distractions such as noise as barriers to their everyday communication. Qualitative research has indicated they feel they "can't cope" (Parr, 2001, p. 276) and have difficulty formulating language (Baylor et al., 2011, p. 278) in distracting or noisy environments. Despite these reports, subjective reactions in response to experimental attention tasks have rarely been addressed. Further examination of how PWA describe their experience communicating in dual task situations may help identify strategies and techniques for meeting attentional demands in everyday communication situations.

We present two complementary studies of how competing attentional demands affect story telling. Study 1 was designed to identify quantitative effects on content accuracy, delivery speed, and perceived effort in 21 participants with aphasia of moderate to mild severity. Study 2 was conducted to explore subjective reactions for the same participants through qualitative methods.

\section{Study 1: Dual task effects on spoken language}

The primary aim of study 1 was to identify dual task effects on perceived effort and spoken language during story retell for participants with moderate or mild aphasia. We predicted 
that PWA would report greater effort than neurotypical controls but that all participants would increase their perceived effort in the dual task condition. We also hypothesized that a dual task condition would cause PWA to retell a story slower and less accurately. Based on previous findings that show decreased speed but maintained accuracy on secondary task performance (Murray, 2000; Murray et al., 1998), we thought neurotypical speakers would only reduce their speed.

\section{Method}

Participants. Thirty-three people participated: Twenty-one had a history of aphasia after stroke $(n=19)$, brain injury $(n=1)$, or multiple sclerosis $(n=1)$ and twelve were control participants with no history of stroke or brain injury. The two participants whose aphasia was not the result of a stroke presented similarly to other PWA due to focal lesions that did not affect nonlinguistic cognitive domains. Three additional participants with aphasia were evaluated but excluded from the study because they were unable to retell a practice story without frequent prompting and cueing from the investigator. This study was approved by the UNC-CH Institutional Review Board.

Participants with aphasia. Participants with aphasia included 8 males and 13 females. The average age of participants was 59 years (range $=32$ to 81 years). All except one had completed at least some college with the average years of education reported as 16 (range $=12$ to 22 years). All participants passed a hearing screening at $40 \mathrm{~dB}$ for $0.5,1$, and $2 \mathrm{KHz}$ or - in the case of two whose hearing was not tested-reported normal hearing. Participants also passed a vision screening, though three were found to have loss to some portion of their right visual field (A04, A08, A16). All were in the chronic stage of aphasia recovery — at least 19 months postonset. 
Participants with aphasia completed a test battery to evaluate their language, verbal working memory, overall cognitive aptitude, and communication confidence. Tests included the Western Aphasia Battery Revised (WAB-R; Kertesz, 2006), the rhyming triplet jud gment and synonym triplet judgment tasks from the Temple Assessment of Language and Short-term memory in Aphasia (TALSA; Martin, Kohen, \& Kalinyak-Fliszar, 2010; Martin, Kohen, Kalinyak-Fliszar, Soveri, \& Laine, 2012), the Test of Nonverbal Intelligence, Fourth Edition (TONI-IV; Brown, Sherbenou, \& Johnson, 2010), and the Communication Confidence Rating Scale for Aphasia (CCRSA; Babbitt, Heinemann, Semik, \& Cherney, 2011; Cherney, Babbitt, Semick, \& Heinemann, 2011). All participants with aphasia were also screened for dysarthria, limb apraxia, and oral apraxia. Clinical test scores and demographic information are reported in Table 1.

We divided the group of participants with aphasia by severity according to the median WAB-R Aphasia Quotient (AQ) score. The moderate group included participants with a score of less than 80 (range $=52.10$ to 77.80 ) and the mild group included all participants with a WAB-R AQ greater than 80 (range $=82.80$ to 100.00 ). Four of these participants scored in the nonaphasic range (i.e., AQ > 93.8); however, they all had a history of aphasia, reported continued word-finding difficulties in their everyday communication, and were judged to have wordfinding errors (e.g., hesitations, circumlocution) during conversation. According to consensus among the first three authors (all experienced speech-language pathologists), ten participants demonstrated concomitant motor speech disorders (apraxia of speech and/or dysarthria). Four of these were participants with mild and six were participants with moderate aphasia. The average age of participants with mild and moderate aphasia was 62 years (range $=33$ to 81 ) and 56 years 
(range $=32$ to 72$)$ respectively. The average years of education was 17 (range $=14$ to 22 ) for participants with mild and 16 (range $=13$ to 20 ) for participants with moderate aphasia.

Control participants. Control participants included five males and seven females. The average age of participants was 58 years (range $=33$ to 81 ) and the average years of education was 16 (range $=12$ to 21$)$. All control participants passed a vision screening. One control participant (C11) reported a congenital hearing problem but was included in the study because he passed a hearing screening at $25 \mathrm{~dB}$ in the left ear $(0.5,1,2,4,6$, and $8 \mathrm{KHz})$ and $45 \mathrm{~dB}$ in the right ear $(0.5,1$, and $2 \mathrm{KHz})$. All other control participants passed a hearing screening at $40 \mathrm{~dB}$ for $0.5,1$, and $2 \mathrm{KHz}$ (Weinstein \& Ventry, 1983). Control participants also completed the Questionnaire to Verify Stroke-free Status (QVSFS; Jones, Williams, \& Meschia, 2001). Their scores and demographic information are shown in Table 2.

Procedures. Participants completed a narrative discourse task under both single and dual task conditions. The stimuli included 4 stories designed to assess narrative production in aphasia (Doyle et al., 1998). These stories were matched for content and complexity (i.e., number of words, number of sentences, number of subord inate clauses and mean sentence length, ratio of clauses to T-units, listening difficulty, and number of unfamiliar words) and are comparable to other commonly used discourse elicitation methods across various measures of verbal productivity, information content, and verbal disruptions (McNeil et al., 2007). Participants were presented with both auditory (i.e., an audio recording of a male speaker telling the story) and visual (i.e., six pictures that went along with the story) stimuli, after which they were asked to retell the story without visual or audio support. Each participant practiced the narrative discourse task by telling one practice story to the investigator before beginning the experiment. The story stimuli were pseudorandomly assigned across conditions. 
Experimental condition. All participants retold a story in a single task and dual task condition. In each condition, the same listener was seated on the other side of a small table. Participants were instructed to retell the story as best they could and include as much detail as possible. During the dual task condition, they simultaneously discriminated between high (2000 $\mathrm{Hz})$ and low $(500 \mathrm{~Hz})$ tones, which were presented pseudo-randomly with an average interstimulus interval of six seconds. Tone presentations lasted $250 \mathrm{~ms}$ and occurred in a sound field over PC speakers with an approximate amplitude of $88 \mathrm{~dB}$. Participants were instructed to push a blue-colored button when they heard the low pitch tone and a red-colored button when they heard the high pitch tone. Prior to the dual task condition, participants practiced identifying tones in isolation for approximately two minutes to ensure they understood the task and to obtain measures of accuracy and response time in isolation. Tones were presented and discrimination accuracy and response times were tracked using a custom Matlab script.

Dependent variables. The main dependent variables were self-rated perceived effort and quantitative story retell performance. Secondary task performance was measured via tone discrimination accuracy and response times in isolation and during the concurrent story retell. Variables and their definitions are listed in Table 3.

Perceived effort. After each story retell, the graduate student left the room and the investigator re-entered and guided the participants in responding to a single question about how difficult they thought the task was (i.e., "Retelling this story was effortful."). The participant circled their answer using a five-point Likert scale ranging from 1 (not at all) to 5 (extremely). A similar measure of speech effort has been used in the stuttering literature (Ingham, Bothe, Jang, Yates, \& Cotton, 2009; Ingham, Warner, Byrd, \& Cotton, 2006). When administered to 
participants with aphasia, the question was read aloud and responses were verified to ensure the participant understood and responded appropriately.

Story retell performance. Primary dependent variables included five measures of spoken language that accounted for content accuracy and delivery speed. The five measures of interest were taken from utterance, word, correct information unit, and disfluency counts. Content was analyzed using broad orthographic transcription in CHAT format (MacWhinney, 2000) and disfluent behaviors were coded in Praat (Boersma \& Weenink, 2014) in order to reference the acoustic signal.

A trained undergraduate research assistant (RA1) and the first author (TH) transcribed the samples in CHAT. Utterance boundaries were identified as outlined in the CHAT manual (MacWhinney, 2000). Word counts obtained from the computerized language analysis (CLAN) software included all intelligible words except fillers or phonological fragments. After the CHAT transcription was completed, RA1 counted correct information units (CIUs; Nicholas \& Brookshire, 1993). CIUs include all words that are intelligible in context, accurate, relevant, and informative. Intrarater and interrater reliability was high for all measures $(r>.80$; see online Supplemental Table S1). Speech productivity was measured in number of CIUs. Speech efficiency was calculated as the ratio of CIUs to words, thus accounting for the proportion of words that contribute to the communicative value of the discourse. Speech rate was calculated in words per minute.

Disfluent behaviors that impede the flow of speech (i.e., simple repetitions, extended pauses, and filled pauses) were coded in Praat, using acoustic segmentation. Repetitions included sound, syllable, and monosyllabic word repetitions. Productions were only coded as repetitions if the same phonemes were produced without any other intervening production. All pauses or filled 
pauses equal to or greater than one second were coded as silent or filled pauses. This was based on previous research showing the mean pause duration in neurotypical speakers to be approximately $1 \mathrm{~s}$ when retelling a story (Kowal, Wiese, \& O’Connell, 1983). In all instances, intrarater and interrater reliability was high $(r>.83$; see online Supplemental Table S1). Repetition and extended pause counts were used to calculate the proportion of repetitions per word and the proportion of extended and filled pauses per utterance.

Statistical Analysis. The effects of complex attention on perceived effort and spoken language for people with moderate, mild, or no aphasia were analyzed using ANOVAs, a Kruskal-Wallis Test, and one-sample t-tests.

Perceived effort, story retell performance, tone discrimination accuracy, and tone discrimination response times (RT) met assumptions of normality and homogeneity of variance and were, therefore, analyzed using two-way mixed effects ANOVAs. The two factors were Group (moderate aphasia, mild aphasia, control) as the between-subject factor and Condition as the within-subject factor. Data distributions did not meet the assumption of homogeneity of variance for repetition and pause data. Logarithmic transformations corrected this problem for pause but not repetition data. Repetition data, therefore, were analyzed using a Kruskal-Wallis Test. Tukey’s HSD or Wilcoxon Rank Sum tests were used to follow up on significant main effects.

A dual task change score was obtained to represent the relative change in performance between single and dual task conditions. This score was calculated by dividing the difference in value between single and dual task performance by the value of single task performance, then multiplying by 100 to express as a percentage (Plummer, Villalobos, Vayda, Moser, \& Johnson, 2014). We will refer to this score as the dual task effect. Negative dual task effects indicate that 
performance deteriorated in the dual task condition (i.e., dual task costs) whereas positive dual task effects indicate that performance improved (i.e., dual task benefits). Dual task effects for each group were analyzed using one-sample t-tests to determine whether performance changed significantly. Differences in dual task effects between participant groups were also analyzed using one-way ANOVAs with Group as the independent variable. Tukey's HSD was used for post-hoc analyses. In addition, change scores were used to visualize and describe dual task effects for individual participants. Finally, correlation analyses were performed to explore relationships between story retell performance and communication confidence, verbal working memory, and nonverbal intelligence.

All statistical analyses were completed using R 3.4.1 (R Core Team, 2017). Mixedeffects ANOVAs were completed on models built using the lme function within the nlme package (Pinheiro, Bates, Debroy, Sarkar, \& R Core Team, 2017) and pairwise comparisons were made on the model using the emmeans package (Lenth, 2017).

\section{Results}

The three participant groups differed in story retell for accuracy (i.e., speech productivity [number of CIUs], efficiency [percent CIUs per word]), and speed (i.e., speech rate [words per minute], repetitions per word, pauses per utterance). Significant group differences were observed for all variables and almost all measure and group pairs. In general, accuracy and speed were lowest for participants with moderate aphasia. Their stories were told with less efficiency, slower speech rate, and more repetitions and pauses than those told by participants with mild and no aphasia. These partipants with moderate aphasia also produced fewer CIUs than participants with

no aphasia. In general, participants with mild aphasia were faster and more accurate than those with moderate aphasia but slower and less accurate than controls. 
In addition to group effects, condition effects on accuracy and speed were noted. In general, the dual task condition reduced story-retell accuracy and speed (see Table 4). A significant main effect of Condition was found for all variables except repetitions. Following an interaction effect, posthoc testing revealed reduced rate during the dual task for the mild and no aphasia group $(p<.001)$ but no change for the moderate aphasia group $(p=.360)$. As shown in Table 5, there was also a reduction in tone discrimination accuracy and increase in RT in the dual task condition. In the following, we first present results related to perceived effort and next present dual task effects on story retell performance.

Perceived effort. We hypothesized that participants would report speaking with greater effort in a dual task condition. Consistent with this hypothesis, analysis of perceived effort ratings showed a main effect for Condition $(F[2,30]=9.366, p=.005)$, revealing greater perceived effort in the dual task condition. Pairwise comparisons following a main effect of Group revealed that participants with mild aphasia reported significantly greater perceived effort than controls when retelling a story $(p=.027)$. The difference between the moderate aphasia group and controls, however, did not reach significance $(p=.066)$. Online Supplemental Figure S2 illustrates these findings.

Story retell performance. All groups were affected by the dual task condition, but the effects varied depending on the group and the measure. First, dual task costs were generally greater for PWA than controls (see Figure 1). Second, dual task costs on accuracy (i.e., productivity, efficiency) were greater for participants with moderate compared with mild aphasia whereas dual task costs on speed (i.e., rate, pauses) were greater for participants with mild compared with moderate aphasia. These findings are discussed in more detail below. Dual task costs on the nonlinguistic tone discrimination task followed a similar pattern with greater 
accuracy reductions for PWA than controls but speed reductions more prominent for the mild than moderate aphasia group. We will first present results related to dual task effects on accuracy followed by results related to dual task effects on speed.

Accuracy. Measures of accuracy included speech productivity (i.e., number of CIUs), which accounts for the number of accurate words produced (i.e., intelligible in context, relevant, informative) and speech efficiency, which accounts for the proportion of words produced that were accurate. Tone discrimination accuracy was also measured as the percent with which participants correctly distinguished between two tones. Dual task effects on accuracy differed among participants with moderate, mild, or no aphasia.

Participants with moderate aphasia experienced prominent dual task costs on tone discrimination and story retell accuracy. Story retell accuracy was reduced in this group both in speech productivity $(t[9]=-4.15, p=.002)$ and efficiency $(t[9]=-2.86, p=.019)$. The dual task cost on both measures was significantly greater for this group than for participants with no aphasia (productivity, $p=.011$; efficiency, $p=.017$ ). Similarly, follow up testing after a significant two-way mixed effects ANOVA showed that participants with moderate aphasia had significantly reduced tone discrimination accuracy $(p=.003)$ whereas those with no aphasia did $\operatorname{not}(p=.642)$.

In contrast, participants with mild aphasia experienced fewer dual task costs on their story retell accuracy. They significantly reduced their speech productivity $(t[10]=-4.44, p=$ $.001)$ but not their efficiency $(t[10]=-.74, p=.474)$. Unlike the moderate aphasia group, differences in dual task costs between participants with mild aphasia and the control group did not reach statistical significance (productivity, $p=.079$; efficiency, $p=.059$ ). Similar to the 
moderate aphasia group, participants with mild aphasia did show significantly reduced tone discrimination accuracy in the dual task condition $(p=.006)$.

The control group showed no dual task effect for story retell accuracy (productivity, $p=$ .746; efficiency, $p=.743$ ). There was also no significant dual task effect on their tone discrimination accuracy $(p=.642)$.

Speed. Measures of speed included speech rate (i.e., words per minute) and pauses (i.e., number of extended and filled pauses per utterance). Tone discrimination RT was also considered a measure of speed. Dual task effects on speed differed among participants with moderate, mild, or no aphasia.

Participants with moderate aphasia experienced few dual task effects on speed. During story retell, they reduced their speech rate $(t[9]=-4.84, p<.001)$ but did not significantly change pauses per utterance $(t[9]=-2.03, p=.073)$. Similarly, they experienced no dual task effect on their tone discrimination $\mathrm{RT}(p=.618)$.

In contrast, participants with mild aphasia and the control group experienced prominent dual task costs on speed during tone discrimination and story retell. Although story retell speed was reduced for both groups, the effect was greater for participants with mild aphasia. Like the control group, participants with mild aphasia experienced dual task costs on their pauses (mild, $p$ $=.0163$; control, $p=.005$ ) and speech rate (mild, $p<.001$; control, $p=.003$ ); however dual task costs on rate were significantly greater for the mild aphasia group $(p=.042)$. Tone discrimination speed in the dual task condition also decreased for participants with mild aphasia and controls. Both groups were slower during dual task tone discrimination (mild, $p=.002$; control, $p<.001)$. 
Correlational analysis. Measures of story retell accuracy and speed were not significantly correlated with communication confidence (i.e., CCRSA scores) or nonverbal intelligence (TONI-IV scores). Verbal working memory (i.e., synonym and rhyming triplet task scores), however, did correlate significantly with story retell accuracy $(p<.01)$ but not speed (see online Supplemental Table S3).

\section{Discussion}

In this quantitative study, we sought to understand how complex attention affects perceived effort and spoken language for people with moderate or mild aphasia. Group-specific speed-accuracy trade-off patterns were observed during story retell. In addition, all participants reported increased perceived effort when retelling a story in the dual task condition, which related to unfavorable changes in most measures of story retell performance. The mild aphasia group reported significantly greater effort than the control group but no difference was found between the moderate aphasia and control groups.

Speed-accuracy trade-offs. Our control group was made up of healthy older adults with no history of brain injury. Older adults require greater processing time than young adults to accurately perform cognitively demanding tasks (Kemper, Herman, \& Lian, 2003). In our study, controls extended their processing time and maintained accuracy in the dual task condition. This confirmed previous reports that healthy older adults slow down on both nonlinguistic (Murray, 2000; Murray et al., 1998) and discourse tasks (Kemper et al., 2003; Oomen \& Postma, 2001) when faced with increased cognitive demands. In addition, our results suggested that, similar to what has been shown with a nonlinguistic task (Murray, 2000; Murray et al., 1998), healthy older adults reduce their delivery speed in order to maintain accuracy during discourse. Similarly, 
participants with mild aphasia maintained relatively good accuracy but greatly reduced their speed.

People with moderate aphasia had the most pronounced dual task costs to accuracy but less costs to speed. It is important to note that speech rate did decrease yet there was no change in their pauses. This suggests that these participants were slowing down but perhaps not pausing at opportune times that would have allowed them to meet the demands of both tasks.

There are several potential explanations for why the moderate aphasia group might have experienced greater dual task costs to accuracy than speed. First, because the processing demands of the story retell task were already great, this group might have been less able to benefit from changes in speed. Even in the single task condition, participants with moderate aphasia were telling stories at a very slow rate (47.92 words per minute) and with many extended and filled pauses (2.07 pauses per utterance). The significant dual task effect on accuracy for the moderate aphasia group might indicate the tendency for attentional demands to interfere more with accuracy for those whose processing of a given task is already slow. In other words, a natural response to increased cognitive demand s might be to decrease speed of performance in order to increase processing time. But if processing time is already high or the demands of the task have already exceeded the speaker's capacity, then slowed processing might reflect more on performance accuracy than speed (see also discussion by Laures, 2005). Given the relationship between verbal working memory and story retell accuracy, it is also plausible that a limited capacity to hold semantic and phonological information in their working memory caused slowing down to be less of a possibility for these participants. Second, more impaired attention might have prevented people with moderate aphasia from using the executive control processes necessary to pause strategically. Although we did not measure attention deficits in this study, 
previous research has demonstrated a correlation between aphasia severity and various standardized measures of attention (Murray, 2012).

Unlike the moderate aphasia group, participants with mild aphasia seemed able to slow down in order to maintain efficiency in the face of increased cognitive demands. Although they generally spoke more slowly and less accurately than controls, they showed a similar speedaccuracy trade-off pattern: maintaining accuracy required participants with mild aphasia to reduce their speech rate significantly more than controls. In addition, people with mild aphasia increased their pausing in the dual task condition. It is possible that in addition to slowing down generally, these participants paused to facilitate attention shifting between the story retell and tone discrimination tasks.

Speech effort during story retell. It is not surprising that under conditions of increased cognitive demands, participants reported greater perceived effort. Indeed, one of the assumptions of the dual task was that it would increase cognitive effort. This finding, nonetheless, confirmed that this was a cognitively demanding condition and confirmed previous research showing that increased task demands result in higher effort ratings for PWA (Murray et al., 1997a).

It may be assumed that production is more effortful for PWA than their peers; previous research, however, has failed to confirm this notion (Clark \& Robin, 1994; Murray et al., 1997a). In the present study, only participants with mild aphasia reported significantly greater effort than the control group. Although the moderate aphasia group generally reported higher perceived effort than controls, they showed much greater variability in these ratings than the mild aphasia group leading to a nonsignificant difference. One explanation for this is that the variability in the moderate aphasia group resulted from a broader spectrum of aphasia types (e.g., more participants with nonfluent aphasia). Upon further inspection of the data, however, this does not 
seem to be the case as low ratings of perceived effort were reported by participants with fluent (e.g., A12) and nonfluent aphasia (e.g., A13). A second potential explanation is that some participants with moderate aphasia did not comprehend the question, leading to inappropriate responses. Although great efforts were made to ensure comprehension and verify responses, this remains a possibility. Because perceived effort was measured using self-report, a third possibility is that participants with moderate aphasia had more difficulty monitoring their effort. A fourth explanation that is consistent with the observed speed-accuracy trade-off is that, due to limited processing resources, some participants with moderate aphasia were unable to expend conscious effort or unaware of their need to do so in the dual task condition, resulting in lower perceived effort ratings and contributing to overall variability. This explanation is in line with previous reports that have interpreted perceived effort ratings as reflecting the amount of cognitive resources expended during a task (Clark \& Robin, 1994).

Speech effort in aphasia has long been of interest and is gaining increased attention; however, methods for measuring effort vary widely. In the present study, we used a self-report measure that allowed us to probe perceived effort relatively quickly, noninvasively, and without specialized equipment. The measure, however, was subjective and-because it was presented through speech and writing - relied somewhat on receptive language skills. Because our sample did not include participants with severe aphasia, we deemed the self-report method appropriate for our study, yet more objective approaches to measuring cognitive effort have been used in recent aphasia research and can be obtained independent of the language modality (e.g., pupillometry, heart rate variability; Chapman \& Hollowell, 2015; Christensen \& Wright, 2014). Future research investigating effort in aphasia should carefully consider which measure is most appropriate. 
In conclusion, study 1 showed that attentional demands interfere with spoken language for PWA. In addition, aphasia severity seemed to affect speed-accuracy trade-off patterns. Similarly, perceived effort increased in the dual task condition but was only significantly greater for the mild aphasia than the control group. Study 2 will expand upon these findings to qualitatively explore how participants reacted to retelling stories in cognitively demanding situations.

\section{Study 2: Subjective Response to a Dual Task}

In study 2, we explored participants' subjective experiences immediately following the experimental protocol from study 1 . Previous qualitative research has investigated the general communicative experiences of PWA (Davidson, Howe, Worrall, Hickson, \& Togher, 2008; Parr, 2007) but, to our knowledge, none have explored their subjective responses following structured and demanding communication experiences that were shared across all participants. We chose qualitative methods to allow participants to share their thoughts about the experience without relying on pre-determined categories. We reasoned that this approach would help us learn about factors that influence everyday communication and how to facilitate successful communication in both clinical and everyday environments. The primary aim of this study was to characterize the subjective experience of PWA when communicating in a dual task condition, which could reflect communication in everyday situations. Parallel to the quantitative study, a secondary aim was to explore whether these experiences varied with aphasia severity.

\section{Method}

We used a qualitative descriptive research design with data derived from semi-structured interviews. Qualitative description is an appropriate method for describing and summarizing specific events experienced by individuals and groups (Lambert \& Lambert, 2012; Sandelowski, 
2000). Like the quantitative study, the qualitative procedures were approved by the UNC-CH Institutional Review Board.

Participants. Participants were the same as those in study 1 with one exception: one control participant (C02) was not included due to a technical problem with the transcription of his interview (see Table 2). This resulted in the inclusion of twenty-one PWA (13 female; 8 male) and eleven age-matched controls ( 7 female; 4 male) with no history of stroke.

Procedure. Immediately after study 1 , participants completed a semi-structured interview. The interview began with questions about the overall experience and then proceeded to discuss the single and dual task conditions. The interviewer also asked probing questions related to participants' feelings including their self-evaluation of their performance retelling the story (see online Supplemental Appendix S4). Consistent with semi-structured interview methodology, the order and wording of questions varied throughout each interview to allow questions to be adapted to the individual needs of each participant (Britten, 1995).

Supported communication strategies were used to ensure comprehension and verify responses, most commonly with the participants who had moderate aphasia. Paper and pen were available to enable graphic and written communication and the interviewer used simple sentences and gestures and wrote key words to support comprehension (Kagan, 1998). When asking participants with auditory comprehension deficits to self-assess their performance the interviewer used a combination of verbal questioning and written choices to elicit and verify responses. Participants with moderate aphasia were also encouraged to use writing to support their responses to interview questions. The papers participants used to support their communication were collected as data and referred to by coders during analysis as supplements to the interview transcripts. Previous studies have used similar supported communication 
strategies during semi-structured interviews (Harmon, Hardy, \& Haley, 2018; Luck \& Rose, 2007).

Analysis. All interviews were transcribed orthographically by a research assistant and paired with the corresponding document for analysis when applicable. Verbatim transcripts were coded in Atlas.ti 8.1.3 using thematic analysis (Braun \& Clarke, 2006). The study employed an eclectic coding process that combined structural, descriptive, emotion, and magnitude coding strategies (Saldaña, 2012).

The first author $(\mathrm{TH})$ and a research assistant (DP) coded the interview transcripts following a five-step iterative process. First, they familiarized themselves with the data by reading all interview transcripts. Second, TH drafted an initial codebook with descriptive codes that were based on his clinical experience, knowled ge of the literature, and purposes of the study (see online Supplemental Appendix S5). Third, TH and DP used the initial codebook to independently code the interview transcripts while taking notes regarding missing, ambiguous, or uninformative codes. Fourth, the coders met to review their respective analyses to discuss discrepancies and refine the codebook. During these discussions, codes were deleted or combined and several subcodes were created to describe the data with greater granularity. For example, codes about emotional reaction were expanded to include emotion subcodes (e.g., frustration, stress, irritation). Fifth, the refined codebook was used to recode the entire set of interview transcripts. This was accomplished by overlaying the new codes and subcodes onto the transcripts that were already marked with the original codes. After recoding, ten discrepancies persisted between the two coders. They were discussed and compared to the codebook definitions to establish consensus about which of the listed codes was most applicable. 
After coding was complete, TH and DP collaboratively organized the codes and subcodes into preliminary themes. After discussing preliminary themes with the fourth author, TH synthesized preliminary themes in relation to the research questions and aims. This resulted in modified themes, subthemes, and categories, which were then presented to and discussed with DP to ensure that they accurately represented the interview data. The final organization of themes and subthemes are presented in Table 6 .

\section{Results}

Analysis of the interview data revealed two themes: "negative reactions to a dual task" and "proactive management of a dual task." Subthemes were nested within each theme with one subtheme being broken down into two categories for further description. We describe each theme with their related subthemes and categories in the following.

Theme I: Negative reactions to a dual task. For PWA, the vast majority of comments about retelling a story with a dual task were negative in nature. In contrast, comments from control participants generally had a positive connotation. Four subthemes were identified: subtheme A addresses negative emotional reactions for PWA in response to the dual task; subtheme B concerns participants' concentration in the dual task condition; subtheme C contrasts the behavioral reactions described by PWA with those of control participants; and subtheme D describes self-evaluation.

Subtheme I.A: Feeling "frustrated," "stress[ed]," and "irritable." PWA experienced feelings of frustration and stress when retelling a story in the dual task condition. Frustration was expressed by four PWA ( 2 moderate, 2 mild). A12 explained that she became "really, really frustrated." Upon recalling their experiences, two PWA threw their hands up in frustration when talking about the difficulty they had. A13 imitated trying to press the buttons while expressing 
her frustration. Participant A19, who had mild aphasia, commented twice on feeling "more stress" in the dual task than the single task condition.

PWA also explained that they became irritated, nervous, or lost their desire to keep trying. When asked about the dual task condition, A06 replied, "it was so irritable!" A10 gestured to indicate that she wanted to get rid of the buttons so she would not have to complete the task. Similarly, three participants with mild aphasia indicated that the dual task weakened their confidence in retelling the story.

One participant with mild aphasia (A18) expressed mixed feelings in response to the dual task. Although she described the task as "hard," she indicated that she was able to remain calm because she knew she "was not going to lose anything by making a mistake." Another participant with mild aphasia explained that he had no emotional reaction to the dual task because "I can't think about a story and pressing buttons and then thinking about how I'm feeling about it." With these exceptions, the emotions reported by PWA were mostly negative, indicating that the dual task invoked frustration or anxiety.

\section{Subtheme I.B: “That took me off task" - losing concentration with a dual task.}

Participants from all groups reported that it was hard to concentrate on both the story retell and tone discrimination tasks and indicated that they had to focus all their energy on the tasks. Some described having to alternate their attention between tasks. For example, A18 reported, "I knew I couldn't talk and press buttons at the same time so I stopped to talk and I know I stopped and then I pressed the buttons and then I would go back.” Although one comment from a control participant went against this trend (C05, “[the dual task] didn't feel that distracting”), all other participants described the dual task condition as interfering with their ability to concentrate. 
Subtheme I.C: Negative behavioral reactions. Participants with aphasia perceived the dual task as having a strong negative effect on their communication behavior whereas control participants perceived minimal effects.

“[My story] was interrupted." Nine PWA (3 moderate, 6 mild) reported feeling unable to do both tasks simultaneously and mentioned "mess[ing] up" on the story, the tone discrimination task, or both. For example, they described their story retell as "interrupted," "cryptic," or prolonged and their tone discrimination as "wrong" or inaccurate.

"I found that I could just tell [the story] and push at the same time." Behavioral reactions to retelling a story with a dual task were rarely mentioned by control participants. Only three controls mentioned an impact on their communication behavior, but they asserted that it was minimal. C03 said that, prior to retelling the story, she was concerned that the dual task might affect her performance negatively, "but then I found I could just tell and push at the same time... I just kind of incorporated it into the story." C09 did not feel like his performance was affected by the dual task but mentioned recognizing that it might be "annoying" for the communication partner. Only one control participant (C07) described any negative behavioral reaction by stating that she felt like her retell with a dual task was less "smooth."

Overall, most PWA perceived the dual task condition to have a negative impact on their communication whereas participants without aphasia perceived the imact to be minimal.

Subtheme I.D: “I was really bad at that" - negative self-assessment. Most PWA gave a negative assessment of their performance when retelling a story with a dual task, whereas most participants without aphasia provided a positive self-assessment. Ten PWA ( 5 moderate, 5 mild $)$ described their performance as "bad," "worse," "hard," "lousy," and "awful." A08 expressed the overall sentiment that represents comments from this group, "I was really bad at that... it was 
awful!" Conversely, participants without aphasia stated that they did "pretty good." One participant (C04) said, "I did well... I felt successful with that and I was very surprised." Contrasting the comments from these groups highlights a difference in how participants with and without aphasia self-assessed their performance in the dual task condition.

Theme II: Proactive management of a Dual Task. Participants with aphasia described different ways in which they managed the increased demands posed by a dual task. This included choices about which task to prioritize while retelling the story and strategies that they intentionally implemented to aid in retelling their story. The majority of these comments were made by participants with mild aphasia. Subtheme A describes how PWA prioritized tasks when retelling a story in the dual task condition. Subtheme B depicts the strategies that were mentioned by participants - particularly those with mild aphasia.

Subtheme II.A: PWA made choices about what to prioritize. Four participants with aphasia explicitly commented on attending to one task more than another during the experiment. Three ( 2 moderate, 1 mild) reported focusing more on the "buttons" and one with mild aphasia (A19) reported concentrating more on the story with less regard for the tone discrimination task.

Subtheme II.B: People with mild aphasia used intentional strategies. Participants with mild aphasia identified a number of strategies that helped them manage the demands of retelling a story with a competing task. These comments were made exclusively by participants with mild aphasia. Over half of them mentioned at least one intentional strategy whereas no participants from the moderate aphasia or control groups commented on a single intentional strategy. The strategies included (1) moving forward, (2) getting it over with, (3) intentional slowing, and (4) rehearsal. 
The most common strategy that participants with mild aphasia discussed was "moving forward." Most often this strategy was identified in relation to moving on with retelling the story regardless of mistakes. In one instance, A04 said, "once I started, [the story] would come easier." He then talked about keeping the story moving forward. A17 moved forward by focusing on the main events or basic structure of the story without getting caught up in smaller details, A08 managed the dual task by "trying to get through [the] story [quickly]," A18 intentionally slowed down and paused so that she could press "the buttons and then... go back" to telling the story, and A19 silently rehearsed specific story details — particularly names - before retelling the story in the dual task condition.

\section{Discussion}

The purpose of this study was to explore the subjective reactions of PWA after retelling a story while simultaneously needing to attend to an external task and to compare those reactions to peers without aphasia. The task elicited negative emotional, behavioral, and evaluative responses from PWA only. Participants with mild aphasia described incorporating strategies to deal with the cognitive challenge, but participants with moderate aphasia did not mention using such strategies. Implications of these findings are discussed below.

Perceived challenges of cognitive demands. Qualitative data from study 2 corroborate quantitative findings from study 1 and previous research, which suggests that producing language with a competing task is particularly challenging for PWA (Baylor et al., 2011; Murray, 2000; Murray et al., 1998; Parr, 2001). This might be the result of a vulnerability that PWA have shown to impairments in attention (Murray, 2012). In addition to confirming the negative behavioral effect of attentional demands on speech and language performance, 
qualitative findings suggest that these negative reactions extend to emotional and evaluative responses.

PWA thought they performed poorly and described experiencing negative emotional reactions. These reactions can be associated with stress (Starcevic \& Castle, 2016) and might intimate a propensity for anxiety in PWA during challenging communication tasks. One hypothesis suggests that language itself invokes anxiety in PWA (termed "linguistic anxiety"; Cahana-Amitay et al., 2011); however, empirical support is lacking for general anxiety associated with language use (see Laures-Gore \& Buchanan, 2015). Alternatively, the heightened anxiety may be more context-specific: An everyday communication situation that is perceived as challenging by neurotypical adults, for example secondary to attentional demands, may be perceived as threatening by PWA, leading to a heightened neurovisceral response (Laures-Gore \& Buchanan, 2015) and potentially affecting spoken language (see e.g., Cahana-Amitay et al., 2015) and life participation. Individual differences related to overall health, energy, and coping resources are likely to mediate the indvididual response (see framework presented in LauresGore \& Buchanan, 2015).

The contrast in how participants with and without aphasia self-assessed their performance also indicates differences in response to cognitive challenges. Theories of motivation suggest that tasks must provide an optimal challenge to support self-efficacy (Bandura, 1997; Ryan \& Deci, 2000). In other words, tasks that are difficult yet achievable will increase confidence in subsequent performances and encourage the actor to persist despite challenges. It appears that the dual task condition provided an optimal challenge for at least some control participants as indicated by the pleasant surprise that $\mathrm{C} 04$ expressed after a performance in this condition that she perceived as acceptable. In contrast, participants with aphasia expressed discomfort with the 
dual task and most perceived their own story retell performance as unacceptable. Had they been offered another chance to complete the task, they would likely have anticipated performing suboptimally again. Similar low self-efficacy in real life communication situations may lead to withdrawal from challenging environments and contribute to the social isolation that has been shown to be prevalent in aphasia (Davidson et al., 2008; Parr, 2007).

Strategies. Notably, all comments about self-selected strategies came from the mild aphasia group. It is possible that participants with moderate aphasia were unable to intentionally employ strategies because the linguistic demands of retelling a story combined with the cognitive demands of the dual task restricted access to attentional resources for this group (see Murray, 2012). If this is the case, explicit strategy training may help people with moderate aphasia improve their performance in demanding communication situations. Another possibility is that expressive language difficulties prevented participants with moderate aphasia from describing the intentional strategies they used. Despite their intact language, however, control participants also did not explicitly comment on intentional strategies. It is possible that moderate aphasia and control participants employed strategies that they did not mention in the interview; nevertheless, the fact that the majority of participants with mild aphasia mentioned at least one strategy compared to none being mentioned by the other two groups is noteworthy. Because most strategies were identified by people with mild aphasia, we will focus our discussion on that group.

Two of the strategies - including the one reported most frequently-encouraged participants to not get hung up on mistakes or details (i.e., "moving forward," "getting it over with"). It seems that people with mild aphasia are aware that overemphasizing errors can detract from the overall message of their story - especially when they are faced with increased 
attentional demands. Similar approaches have been used therapeutically to help PWA with varied levels of severity relay a message without getting derailed by linguistic and phonetic errors (e.g., script training [Cherney, Halper, Holland, \& Cole, 2008]; integral stimulation [Fridriksson, Basilakos, Hickok, Bonilha, \& Rorden, 2015; Wambaugh, West, \& Doyle, 1998]) and may have relatively immediate application to real life settings. In contrast, more resourcedemanding strategies (e..g., implicit "rehearsal") were mentioned less often. Intervention methods that emphasize correcting impaired behavior in the context of discourse (Boyle, 2011; Murray, Timberlake, \& Eberle, 2007) may be more senstive to attentional demands, including transfer to real-life communication situations. A better understanding of how training and communication strategies transfer outside the attentionally supportive therapy environment would help tailor therapies to strategies that might better improve everyday communication. Attention to how people with aphasia describe strategies that help them communicate in challenging situations may guide the selection of customized intervention procedures for this group (Haley, Cunningham, de Riesthal, \& Barry, in press; Harmon et al., 2018).

\section{Conclusions}

Everyday communication most often occurs in situations where attentional demands are high. Distractions such as background noise and others talking might divide a speaker's attention from the communication task (Baylor et al., 2011; Dalemans et al., 2010; Parr, 2001). The purpose of the present work was to investigate how complex attentional demands affect retelling a story for PWA as measured objectively by the content and speed of their spoken language and subjectively by their personal reports of the communication experience. Taken together, the two studies showed that communicating with a competing task is particularly difficult for PWA and 
that their response to dual task communication is affected by the severity of the language impairment.

Combined quantitative and qualitative findings showed that a dual task had general and aphasia-specific effects. In general, all participants increased their effort in the dual task condition. This was shown by significantly higher ratings of perceived effort following the dual task condition as well as participants' explanations that it required all their energy. For PWA, this increase in perceived effort met with performance decrements as well as negative emotional reactions, the nature of which was influenced by aphasia severity.

Aphasia severity seemed to play a role in how participants responded to retelling a story in a dual task condition. This was seen quantitatively through group specific speed-accuracy trade-off patterns and qualitatively through increased comments by participants with mild aphasia about actively responding to the dual task. Specifically, study 1 showed decreased speech rate and increased pausing for the mild aphasia group in the dual task condition, which aligned somewhat with the intentional strategies they discussed in study 2 . In particular, the strategy of "moving forward" implies that participants were aware at some level of the slowed and broken nature of their story retell in this condition. One participant specifically mentioned slowing down in order to deal with the demands of the dual task. The fact that these strategies were mentioned by participants with mild and not moderate aphasia suggests a greater ability to cope with cognitive demands for this group.

While it appears that people with mild aphasia may be better equipped to confront cognitively demanding communication situations than those with moderate aphasia, explicit training might help them meet these demands during everyday communication. For example, therapy for people with mild aphasia could incorporate practice in dual task situations to simulate 
real-world distractions and facilitate generalization (see e.g., Hinckley, Patterson, \& Carr, 2001). Given the results of the present study, a suggested approach to such training would be to first emphasize accuracy (allowing the client to decrease speed as necessary) then incrementally help the client increase speed while maintaining accuracy. Recently, a similar approach in wordfinding treatment showed better contextual generalization than focusing on accuracy only (Conroy, Drosopoulou, Humphreys, Halai, \& Lambon Ralph, 2018). Therapy might also integrate explicit instruction and practice pertaining to slowing down when situational demands increase.

In sum, the results indicate that cognitively demanding communication situations pose greater challenge for PWA than neurotypical speakers and that aphasia severity impacts how people cope with these demands. Incorporating cognitive demands into therapy might be more appropriate for people with mild aphasia than those with more severe impairment. Such training should be designed to optimize self-efficacy and reduce anxiety.

Acknowledgments: This work was supported by a Dissertation Completion Fellowship Award from the University of North Carolina Graduate School. Special thanks to Daniel Picetti, Marissa Rock, Laura Blizard, Madison Goers, Jenna Hall, Emmie Costen, Tayler Simonds, Morgan Billinger, and Matthew Suderman for their help with data collection and analysis. We also wish to thank the Triangle Aphasia Project, unlimited for their help with recruitment and Dr. Christopher Dromey for his feedback on an earlier version of the manuscript. 


\section{References}

Bandura, A. (1997). Self-Efficacy: The Exercise of Control. New York: W. H. Freeman and Company.

Baylor, C., Burns, M., Eadie, T., Britton, D., \& Yorkston, K. (2011). A qualitative study of interference with communicative participation across communication disorders in adults. American Journal of Speech-Langauge Pathology, 20(November), 269-287. http://doi.org/10.1044/1058-0360(2011/10-0084)intervention

Boersma, P., \& Weenink, D. (2014). Praat: Doing phonetics by computer. Retrieved from http://www.praat.org/

Braun, V., \& Clarke, V. (2006). Using thematic analysis in psychology. Qualitative Research in Psychology, 3(2), 77-101. http://doi.org/10.1191/1478088706qp063oa

Cahana-Amitay, D., Albert, M. L., Pyun, S.-B., Westwood, A., Jenkins, T., Wolford, S., \& Finley, M. (2011). Language as a stressor in aphasia. Aphasiology, 25(2), 593-614. http://doi.org/10.1080/02687038.2010.541469

Cahana-Amitay, D., Oveis, A. C., Sayers, J. T., Pineles, S. L., Spiro, A., \& Albert, M. L. (2015). Biomarkers of "Linguistic Anxiety" in aphasia: A proof-of-concept case study. Clinical Linguistics \& Phonetics, 29(5), 401-413. http://doi.org/10.3109/02699206.2015.1014572

Chapman, L. R., \& Hollowell, B. (2015). A novel pupillometric method for indexing word difficulty in individuals with and without aphasia. Journal of Speech, Language, and Hearing Research, 58(October), 1508-1520. http://doi.org/10.1044/2015

Christensen, S. C., \& Wright, H. H. (2014). Quantifying the effort individuals with aphasia invest in working memory tasks through heart rate variability. American Journal of SpeechLanguage Pathology, 23(May), 361-371. http://doi.org/10.1044/2014

Conroy, P., Drosopoulou, C. S., Humphreys, G. F., Halai, A. D., \& Lambon Ralph, M. A. (2018). Time for a quick word? The striking benefits of training speed and accuracy of word retrieval in post-stroke aphasia. Brain, 141, 1815-1827.

http://doi.org/10.1093/brain/awy087

Dalemans, R. J. P., de Witte, L., Wade, D., \& van den Heuvel, W. (2010). Social participation through the eyes of people with aphasia. International Journal of Language \& Communication Disorders, 45(5), 537-550. http://doi.org/10.3109/13682820903223633

Davidson, B., Howe, T., Worrall, L., Hickson, L., \& Togher, L. (2008). Social participation for older people with aphasia: The impact of communication disability on friend ships. Topics in Stroke Rehabilitation, 15(4), 325-340. http://doi.org/10.1310/tsr1504-325

Garcia, L. J., Barrette, J., \& Laroche, C. (2000). Perceptions of the obstacles to work reintegration for persons with aphasia. Aphasiology, 14(3), 269-290. http://d oi.org/10.1080/026870300401478 
Haley K.L., Cunningham, K., Barry J., \& de Riesthal M. (In press). Setting goals for communicative life participation in aphasia. American Journal of Speech-Language Pathology.

Harmon, T. G., Hardy, L., \& Haley, K. L. (2018). Proactive social validation of methods and procedures used for training speech production in aphasia. Aphasiology, 32(8), 922-943. http://doi.org/10.1080/02687038.2017.1385051

Hinckley, J. J., \& Carr, T. H. (2005). Comparing the outcomes of intensive and non-intensive context-based aphasia treatment. Aphasiology, 19(10-11), 965-974. http://d oi.org/10.1080/02687030544000173

Hinckley, J. J., Patterson, J. P., \& Carr, T. H. (2001). Differential effects of context- and skillbased treatment approaches: Preliminary findings. Aphasiology, 15(5), 463-476. http://d oi.org/10.1080/02687040042000340

Hula, W. D., \& McNeil, M. R. (2008). Models of attention and dual-task performance as explanatory constructs in aphasia. Seminars in Speech and Language, 29(3), 169-187. http://doi.org/10.1055/s-0028-1082882

Hula, W. D., McNeil, M. R., \& Sung, J. E. (2007). Is there an impairment of language-specific attentional processing in aphasia? Brain and Language, 103(1-2), 240-241. http://doi.org/10.1016/j.bandl.2007.07.023

Ingham, R. J., Bothe, A. K., Jang, E., Yates, L., \& Cotton, J. (2009). Measurement of speech effort during fluency-inducing conditions in adults who do and do not stutter. Journal of Speech, Language, and Hearing Research, 52(October), 1286-1301.

Ingham, R. J., Warner, A., Byrd, A., \& Cotton, J. (2006). Speech effort measurement and stuttering: Investigating the chorus reading effect. Journal of Speech, Language, and Hearing Research, 49(3), 660-670. http://doi.org/10.1044/1092-4388(2006/048)

Jones, W. J., Williams, L. S., \& Meschia, J. F. (2001). Validating the Questionnaire for Verifying Stroke-Free Status (QVSFS) by neurological history and examination. Stroke, 32(10), 2232-2236. http://doi.org/10.1161/hs1001.096191

Kagan, A. (1998). Supported conversation for adults with aphasia: Methods and resources for training conversation partners. Aphasiology, 12(9), 816-830.

Kemper, S., Herman, R. E., \& Lian, C. H. T. (2003). The costs of doing two things at once for young and older adults: Talking while walking, finger tapping, and ignoring speech or noise. Psychology and Aging, 18(2), 181-192. http://doi.org/10.1037/0882-7974.18.2.181

Kemper, S., McDowd, J., Pohl, P., Herman, R., \& Jackson, S. (2006). Revealing language deficits following stroke: The cost of doing two things at once. Aging, Neuropsychology, and Cognition, 13(1), 115-139. http://doi.org/10.1080/13825580500501496

Kowal, S., Wiese, R., \& O’Connell, D. C. (1983). The use of time in storytelling. Language and 
Speech, 26(4), 377-392.

Kurland, J. (2011). The role that attention plays in language processing. Perspectives on Neurophysiology and Neurogenic Speech and Language Disorders, 21(2), 44-77. https://doi.org/10.1044/nnsld21.2.47

Kreindler, A., \& Fradis, A. (1968). Performance in aphasia: A neurodynamical diagnostic and psychological study. Paris: Gauthier-Vollars.

Lambert, V. A., \& Lambert, C. E. (2012). Editorial: Qualitative descriptive research: An acceptable design. Journal of Nursing Research, 16(4), 255-256.

LaPointe, L. L., \& Erickson, R. J. (1991). Auditory vigilance during divided task attention in aphasic individuals. Aphasiology, 5(6), 511-520.

http://doi.org/10.1080/02687039108248556

Laures-Gore, J. S., \& Buchanan, T. W. (2015). Aphasia and the neuropsychobiology of stress. Journal of Clinical and Experimental Neuropsychology, 37(7), 688-700. http://doi.org/10.1080/13803395.2015.1042839

Laures, J. S. (2005). Reaction time and accuracy in individuals with aphasia during auditory vigilance tasks. Brain and Language, 95(2), 353-357. http://doi.org/10.1016/j.bandl.2005.01.011

Le Dorze, G., Salois-Bellerose, É., Alepins, M., Croteau, C., \& Hallé, M.-C. (2014). A description of the personal and environmental determinants of participation several years post-stroke according to the views of people who have aphasia. Aphasiology, 28(4), 421439. http://doi.org/10.1080/02687038.2013.869305

Lenth, R. (2017). emmeans: Estimated Marginal Means, aka Least-Squares Means.

Luck, A. M., \& Rose, M. L. (2007). Interviewing people with aphasia: Insights into method adjustments from a pilot study. Aphasiology, 21(2), 208-224. http://doi.org/10.1080/02687030601065470

MacWhinney, B. (2000). The CHILDES Project: Tools for Analyzing Talk (3rd ed.). Mahway, NJ: Lawrence Erlbaum Associates.

Martin, N., Kohen, F., \& Kalinyak-Fliszar, M. (2010). A processing approach to the assessment of language and verbal short-term memory abilitites in aphasia. In Clinical Aphasiology Conference. Charleston, SC. http://doi.org/10.1080/02687038.2010.496261

Martin, N., Kohen, F., Kalinyak-Fliszar, M. M., Soveri, A., \& Laine, M. (2012). Effects of working memory load on processing of sounds and meanings of words in aphasia. Aphasiology, 26(3-4), 462-493. http://doi.org/10.1080/02687038.2011.619516

McNeil, M. R., Odell, K., \& Tseng, C.-H. (1991). Toward the integration of resource allocation 
into a general theory of aphasia. In Clinical Aphasiology Conference (pp. 21-39). Santa Fe, NM.

Murray, L. L. (2000). The effects of varying attentional demands on the word retrieval skills of adults with aphasia, right hemisphere brain damage, or no brain damage. Brain and Language, 72, 40-72. http://doi.org/10.1006/brln.1999.2281

Murray, L. L. (2002). Attention deficits in aphasia: Presence, nature, assessment, and treatment. Seminars in Speech and Language, 23(2), 107-116. http://doi.org/10.1055/s-2002-24987

Murray, L. L. (2012). Attention and other cognitive deficits in aphasia: Presence and relation to language and communication measures, 21(May), 51-64. http://doi.org/10.1044/10580360(2012/11-0067)

Murray, L. L., Holland, A. L., \& Beeson, P. M. (1997a). Accuracy monitoring and task demand evaluation in aphasia. Aphasiology, 11(4-5), 401-414. http://d oi.org/10.1080/02687039708248480

Murray, L. L., Holland, A. L., \& Beeson, P. M. (1997b). Auditory processing in individuals with mild aphasia: a study of resource allocation. Journal of Speech, Language \& Hearing Research, 40(4), 792-808.

Murray, L. L., Holland, A. L., \& Beeson, P. M. (1998). Spoken language of individuals with mild fluent aphasia under focused and divided-attention conditions. Journal of Speech Language and Hearing Research, 41(1), 213-227.

Murray, L. L., Timberlake, A., \& Eberle, R. (2007). Treatment of underlying forms in a discourse context. Aphasiology, 21(2), 139-163. http://doi.org/10.1080/02687030601026530

Navon, D., \& Miller, J. (2002). Queuing or sharing? A critical evaluation of the single-bottleneck notion. Cognitive Psychology, 44(3), 193-251. http://doi.org/10.1006/cogp.2001.0767

Oomen, C. C., \& Postma, A. (2001). Effects of divided attention on the production of filled pauses and repetitions. Journal of Speech, Language, and Hearing Research, 44(5), 9971004. http://doi.org/10.1044/1092-4388(2001/078)

Parr, S. (2001). Psychosocial aspects of aphasia: Whose perspectives? Folia Phoniatrica et Logopaedica, 53(5), 266-88. http://doi.org/52681

Parr, S. (2007). Living with severe aphasia: Tracking social exclusion. Aphasiology, 21(1), 98123. http://doi.org/10.1080/02687030600798337

Pinheiro, J., Bates, D., Debroy, S., Sarkar, D., \& R Core Team (2017). nlme: Linear and nonlinear mixed effects models. Retrieved from https://cran.r-project.org/package=nlme 
Plummer, P., Villalobos, R. M., Vayda, M. S., Moser, M., \& Johnson, E. (2014). Feasibility of dual-task gait training for community-dwelling adults after stroke: A case series. Stroke Research and Treatment, 2014. http://doi.org/10.1155/2014/538602

Ryan, R. M., \& Deci, E. L. (2000). Intrinsic and extrinsic motivations: Classic definitions and new directions. Contemporary Educational Psychology, 25(1), 54-67. http://d oi.org/10.1006/ceps.1999.1020

Saldaña, J. (2012). The Coding Manual for Qualitative Researchers (2nd ed.). SAGE Publications.

Sandelowski, M. (2000). Whatever Happened to Qualitative Description? Research in Nursing \& Health, 23, 334-340. http://doi.org/10.1002/1098-240x(200008)23:4<334::aidnur9>3.0.co;2-g

Starcevic, V., \& Castle, D. J. (2016). Anxiety Disorders. In Stress: Concepts, Cognition, Emotion, and Behavior (pp. 203-211). Academic Press. http://doi.org/10.1016/B978-0-12800951-2.00024-8

R Core Team (2017). R: A language and environment for statistical computing. Vienna, Austria: R Foundation for Statistical Computing. Retrieved from http://www.r-project.org/

Tseng, C. H., McNeil, M. R., \& Milenkovic, P. (1993). An investigation of attention allocation deficits in aphasia. Brain and Language. http://doi.org/10.1006/brln.1993.1046

Weinstein, B. E., \& Ventry, I. M. (1983). Audiometric correlates of the hearing handicap inventory for the elderly. Journal of Speech and Hearing Disorders, 48(November), 379384. 
Table 1. Information for Participants with Aphasia.

\begin{tabular}{|c|c|c|c|c|c|c|c|c|c|c|c|c|c|c|}
\hline \multirow[t]{2}{*}{ ID } & \multirow[t]{2}{*}{ Sex } & \multirow[t]{2}{*}{ Age } & \multirow[t]{2}{*}{ Education } & \multirow{2}{*}{$\begin{array}{c}\text { TPO } \\
\text { (yy;mm) }\end{array}$} & \multirow[t]{2}{*}{ Etiology } & \multicolumn{2}{|c|}{ WAB-R } & \multicolumn{2}{|c|}{ TALSA } & \multirow[t]{2}{*}{ TONI-IV } & \multirow[t]{2}{*}{ CCRSA } & \multicolumn{3}{|c|}{ Screenings } \\
\hline & & & & & & $A Q$ & Classification & $\begin{array}{l}\text { Rhyming } \\
\text { Total (\%) }\end{array}$ & $\begin{array}{l}\text { Synonym } \\
\text { Total (\%) }\end{array}$ & & & Dysarthria & $\begin{array}{l}\text { Limb } \\
\text { Apraxia }\end{array}$ & $\begin{array}{l}\text { Oral } \\
\text { Apraxia }\end{array}$ \\
\hline $\mathrm{A} 01$ & $\mathrm{~F}$ & 58 & 20 & $14 ; 00$ & Stroke & 87.00 & Anomic & 76.67 & 80.00 & 92 & 72 & 8 & 31 & 21 \\
\hline $\mathrm{A} 02$ & $\mathrm{~F}$ & 56 & 14 & $4 ; 05$ & Stroke & 90.70 & Anomic & 83.33 & 72.50 & 88 & 63 & 11 & 48 & 39 \\
\hline A03 & $\mathrm{F}$ & 81 & 15 & $5 ; 07$ & Stroke & 92.70 & Anomic & 86.67 & 87.50 & 94 & 55 & 8.5 & 39 & 39 \\
\hline A04 & $M$ & 33 & 16 & $5 ; 11$ & $\mathrm{TBI}$ & 87.60 & Anomic & 56.67 & 62.50 & 92 & 90 & 10.5 & 50 & 44 \\
\hline A06 & $\mathrm{F}$ & 48 & 17 & $18 ; 04$ & MS & 77.80 & $\begin{array}{l}\text { Transcortical } \\
\text { Motor }\end{array}$ & 76.67 & 85.00 & 95 & 76 & 12.5 & 50 & 50 \\
\hline A08 & $\mathrm{M}$ & 56 & 16 & $2 ; 01$ & Stroke & 100.00 & NABW & 100.00 & 97.50 & 128 & 78 & 12.5 & 50 & 48 \\
\hline A09 & $\mathrm{F}$ & 59 & 16 & $1 ; 10$ & Stroke & 89.90 & Anomic & 80.00 & 90.00 & 88 & 80 & 13 & 50 & 47 \\
\hline A10 & $\mathrm{F}$ & 72 & 12 & $8 ; 00$ & Stroke & 72.20 & Broca & 76.67 & 75.00 & 103 & 84 & 9 & 46 & 32 \\
\hline A11 & $\mathrm{F}$ & 65 & 15 & $7 ; 07$ & Stroke & 84.30 & Anomic & 80.00 & 95.00 & 119 & 84 & 10 & 50 & 44 \\
\hline A12 & $\mathrm{F}$ & 61 & 16 & $11 ; 03$ & Stroke & 74.10 & Anomic & 80.00 & 80.00 & 90 & 78 & 10.5 & 27 & 27 \\
\hline A13 & $\mathrm{F}$ & 61 & 13 & $5 ; 04$ & Stroke & 67.50 & Broca & 73.33 & 62.50 & 101 & 79 & 10 & 44 & 32 \\
\hline A14 & $\mathrm{M}$ & 61 & 20 & $5 ; 08$ & Stroke & 67.00 & Wernicke's & 33.33 & 72.50 & 93 & 55 & 13 & 47 & 42 \\
\hline A16 & $M$ & 60 & 22 & $4 ; 08$ & Stroke & 95.40 & NABW & 83.33 & 92.50 & 107 & 94 & 12 & 50 & 49 \\
\hline A17 & $\mathrm{F}$ & 72 & 18 & $9 ; 09$ & Stroke & 82.80 & Anomic & 70.00 & 87.50 & 95 & 81 & 9.5 & 50 & 29 \\
\hline A18 & $\mathrm{F}$ & 71 & 16 & $18 ; 01$ & Stroke & 94.00 & NABW & 96.67 & 97.50 & 104 & 70 & 13 & 50 & 50 \\
\hline A19 & $\mathrm{M}$ & 72 & 18 & $8 ; 02$ & Stroke & 97.40 & NABW & 100.00 & 97.50 & 95 & 72 & 12 & 49 & 41 \\
\hline A20 & M & 60 & 18 & $8 ; 07$ & Stroke & 75.00 & Anomic & 66.67 & 85.00 & 95 & 74 & 13 & 47 & 43 \\
\hline A21 & $\mathrm{F}$ & 32 & 13 & $11 ; 11$ & Stroke & 63.70 & Conduction & 30.00 & 50.00 & 95 & 99 & 10 & 50 & 34 \\
\hline A22 & $\mathrm{F}$ & 56 & 16 & $12 ; 09$ & Stroke & 52.10 & Broca & 30.00 & 60.00 & 105 & 67 & 11 & 44 & 43 \\
\hline A23 & M & 64 & 18 & $3 ; 02$ & Stroke & 72.70 & Broca & 70.00 & 65.00 & 95 & 75 & 8.5 & 48 & 35 \\
\hline A24 & $M$ & 48 & 19 & $1 ; 07$ & Stroke & 68.20 & Broca & 83.33 & 90.00 & 109 & 86 & 11 & 47 & 45 \\
\hline
\end{tabular}

Note. Age and Education are reported in years. TPO = time post-onset; TONI-IV $=$ Test of Nonverbal Intelligence $4^{\text {th }}$ Edition; WAB-R $=$ Western Aphasia Battety-Revised; $A Q=$ Aphasia Quotient; NABW = not aphasic by WAB-R; TALSA = Temple Assessment of Language processing and Short-term memory in Aphasia; GDS = Geriatric Depression Scale; CCRSA = Communication Confidence Rating Scale. Lower dysarthria, limb apraxia, and oral apraxia ratings indicate greater pres ence of dysarthria or apraxia respectively. ${ }^{*}$ Data from this participant were only included in study 2 due to modifications required on the discourse production task. 
Table 2. Control Participant Information.

\begin{tabular}{llrcc}
\hline ID & Sex & Age & Education & QVSFS \\
\hline C01 & F & 71 & 16 & 0 \\
C02* & M & 70 & 21 & 0 \\
C03 & F & 50 & 16 & 0 \\
C04 & F & 55 & 13 & 0 \\
C05 & F & 33 & 18 & 0 \\
C06 & F & 81 & 18 & 0 \\
C07 & F & 34 & 16 & 0 \\
C08 & F & 60 & 12 & 0 \\
C09 & M & 64 & 18 & 0 \\
C10 & M & 66 & 18 & 0 \\
C11 & M & 48 & 14 & 0 \\
C12 & M & 61 & 16 & 1 \\
\hline
\end{tabular}

Note. Age and Education are reported in years.

QVSFS = Questionnaire for Verifying Stroke-Free Status. *Data from this participant were only included in study 1 due to a technical problem with the interview transcription. 
Table 3. Dependent Variables and Their Associated Definitions.

\begin{tabular}{|c|c|c|}
\hline Construct & Variable Name & Definition \\
\hline Effort & Perceived Effort & Self-rating of effort \\
\hline \multirow[t]{3}{*}{ Content Accuracy } & Productivity & Number of Correct Information \\
\hline & & $\begin{array}{l}\text { Units (i.e., intelligible, accurate, } \\
\text { relevant, and informative words) }\end{array}$ \\
\hline & Efficiency & $\begin{array}{l}\text { Ratio of Correct Information Units } \\
\text { per word }\end{array}$ \\
\hline \multirow[t]{3}{*}{ Delivery Speed } & Rate & Number of words per minute \\
\hline & Repetitions & $\begin{array}{l}\text { Proportion of sound, syllable, and } \\
\text { monosyllabic word repetitions per } \\
\text { word }\end{array}$ \\
\hline & Pauses & $\begin{array}{l}\text { Ratio of extended and filled pauses } \\
\text { per utterance }\end{array}$ \\
\hline \multirow{3}{*}{$\begin{array}{l}\text { Secondary Task } \\
\text { Performance }\end{array}$} & Tone Discrimination Accuracy & Percentage of tones accurately \\
\hline & & identified as high or low \\
\hline & $\begin{array}{l}\text { Tone Discrimination Response } \\
\text { Time }\end{array}$ & $\begin{array}{l}\text { Time between the tone offset and } \\
\text { accurate button press }\end{array}$ \\
\hline
\end{tabular}


Table 4. Descriptive Data for Story Retell Performance Among Participant Groups Within Three Experimental Conditions.

\begin{tabular}{|c|c|c|c|c|c|c|c|}
\hline & & \multicolumn{2}{|c|}{ Moderate Aphasia } & \multicolumn{2}{|c|}{ Mild Aphasia } & \multicolumn{2}{|c|}{ Control } \\
\hline & & ST & DT & ST & DT & ST & DT \\
\hline \multirow[t]{4}{*}{ Productivity } & $M$ & 43.50 & 29.50 & 108.82 & 72.09 & 195.00 & 180.92 \\
\hline & $S D$ & 32.88 & 37.44 & 44.97 & 28.18 & 80.66 & 81.74 \\
\hline & Median & 36.00 & 18.50 & 106.00 & 74.00 & 169.00 & 165.00 \\
\hline & Range & $13-122$ & $4-128$ & $38-207$ & $37-137$ & $116-426$ & $122-428$ \\
\hline \multirow[t]{4}{*}{ Efficiency } & $M$ & 36.84 & 24.38 & 62.37 & 55.15 & 87.36 & 87.71 \\
\hline & $S D$ & 18.62 & 14.85 & 19.98 & 16.13 & 8.59 & 7.24 \\
\hline & Median & 33.28 & 26.96 & 61.63 & 58.67 & 89.05 & 89.16 \\
\hline & Range & $17.92-72.22$ & $2.70-46.90$ & $22.75-95.74$ & $27.21-73.15$ & 70.04-97.48 & 64.49-98.17 \\
\hline \multirow[t]{4}{*}{ Rate } & $M$ & 47.92 & 38.92 & 102.16 & 76.93 & 158.52 & 137.68 \\
\hline & $S D$ & 19.51 & 19.99 & 31.53 & 27.15 & 22.33 & 19.17 \\
\hline & Median & 56.19 & 41.12 & 110.00 & 82.45 & 156.72 & 144.24 \\
\hline & Range & $14.09-71.87$ & $8.98-65.07$ & $41.30-152.91$ & $28.60-122.10$ & $115.97-204.15$ & $105.68-157.33$ \\
\hline \multirow[t]{4}{*}{ Repetitions } & $M$ & 0.03 & 0.03 & 0.02 & 0.01 & 0.00 & 0.01 \\
\hline & $S D$ & 0.02 & 0.02 & 0.02 & 0.01 & 0.01 & 0.01 \\
\hline & Median & 0.03 & 0.02 & 0.02 & 0.01 & 0.00 & 0.01 \\
\hline & Range & $.01-.06$ & $.00-.07$ & $.01-.06$ & $.00-.05$ & $.00-.02$ & $.00-.02$ \\
\hline \multirow[t]{4}{*}{ Pauses } & $M$ & 2.07 & 2.75 & 0.77 & 1.25 & 0.49 & 0.72 \\
\hline & $S D$ & 0.75 & 1.29 & 0.33 & 0.71 & 0.37 & 0.39 \\
\hline & Median & 2.18 & 2.83 & 0.73 & 1.25 & 0.41 & 0.70 \\
\hline & Range & $.69-2.92$ & $.73-5.22$ & $.20-1.50$ & $.42-2.55$ & $.11-1.13$ & $.09-1.30$ \\
\hline
\end{tabular}

Note. SP = Supportive Partner (i.e., communication partner demonstrating supportive nonverbal feedback); NP = Nonsupportive Partner (i.e., communication partner demonstrating nonsupportive nonverbal feedback); DT = Dual Task (i.e., concurrent tone discrimination). 
Table 5. Means and Standard Deviations for Tone Discrimination Accuracy and Response Time in Isolation and while Concurrently Retelling a Story Across Three Groups.

\begin{tabular}{|c|c|c|c|c|}
\hline & \multicolumn{2}{|c|}{ Isolation } & \multicolumn{2}{|c|}{ With Discourse } \\
\hline & Accuracy & $\mathrm{RT}(\mathrm{s})$ & Accuracy & $\mathrm{RT}(\mathrm{s})$ \\
\hline Moderate Aphasia & $0.88(.16)$ & $1.11(.42)$ & $0.61(.28)$ & $1.28(.29)$ \\
\hline Mild Aphasia & $0.92(.10)$ & $0.87(.20)$ & $0.68(.26)$ & $1.32(.30)$ \\
\hline Control & $0.99(.02)$ & $0.82(.14)$ & $0.90(.03)$ & $1.45(.40)$ \\
\hline
\end{tabular}

Note. Only accurate button press responses were included in the average response time (RT) measures. 
Table 6. Organizational Structure of Themes, Subthemes, and Categories.

Themes and subthemes

Categories

\section{Negative reactions to a dual task}

A. Feeling "frustrated," "stress[ed]," and

"irritatable."

B. "That took me off task" - losing concentration with a dual task.

C. Negative behavioral reactions

1. "[My story] was interrupted"

2. "I found that I could just tell [the story] and push at the same time"

D. "I was really bad at that" - negative selfassessment

\section{Proactive management of a dual task}

A. PWA made choices about what to prioritize

B. People with mild aphasia used intentional strategies 
Figure 1. Dual task effects on measures of accuracy (productivity and efficiency) and speed (rate and pauses) during story retell production across participant groups. A negative change represents dual task costs. Asterisks above bars show significant dual task costs on that measure for the specified group. Bracketed asterisks show significant differences in dual task costs between the specified groups. Mod = moderate aphasia group; Mild = mild aphasia group; Control $=$ control group. Error bars indicate stand ard error. ${ }^{*} p<.05 ; * * p<.01 ; * * *<.001$.

Supplemental Table S1. Intrarater and interrater reliability for spoken language codes.

Supplemental Figure S2. Perceived effort ratings among participants with mild, moderate, or no aphasia in single task and dual task conditions.

Supplemental Table S3. Correlations between assessment scores and story retell accuracy and speed.

Supplemental Appendix S4. Semi-structured interview guide.

Supplemental Appendix S5. Codes and abridged codebook definitions used to analyze interview data and assign meaning units together into themes and subthemes. 